\title{
Presence of cancer-associated fibroblasts inversely correlates with Schwannian stroma in neuroblastoma tumors
}

\author{
Rana Zeine ${ }^{1}$, Helen R Salwen ${ }^{2}$, Radhika Peddinti ${ }^{2}$, Yufeng Tian ${ }^{2}$, Lisa Guerrero ${ }^{2}$, \\ Qiwei Yang ${ }^{2}$, Alexandre Chlenski ${ }^{2}$ and Susan L Cohn ${ }^{2}$ \\ ${ }^{1}$ Department of Pathology, Northwestern University, Chicago, IL, USA and ${ }^{2}$ Department of Pediatrics, \\ University of Chicago, Chicago, IL, USA
}

\begin{abstract}
Stromal cells have a central function in the regulation of tumor angiogenesis. Recent studies have shown that stromal myofibroblasts (cancer-associated fibroblasts) actively promote tumor growth and enhance tumor angiogenesis in many types of adult carcinomas. To evaluate the function cancer-associated fibroblasts have in neuroblastoma angiogenesis and investigate their relationship to stromal Schwann cells, we quantified cancerassociated fibroblasts in 60 primary neuroblastoma tumors and in a novel neuroblastoma xenograft model in which murine Schwann cells were induced to infiltrate into the tumor stroma. Tumor sections were examined for presence of microvascular proliferation, a hallmark of tumor angiogenesis. Cancer-associated fibroblasts were characterized by positive immunostaining for $\alpha$-smooth muscle actin $(\alpha-$ SMA) and were distinguished from pericytes by staining negatively for high-molecular-weight caldesmon. $\alpha$-SMA-positive cells were quantified and their number was defined as high when $>1.0 \%$ of the area was positive. Associations between high cancerassociated fibroblast number, microvascular proliferation and established prognosticators were analyzed. High numbers of cancer-associated fibroblasts were associated with Schwannian stroma-poor histopathology and microvascular proliferation. Thirty-seven ( $80 \%$ ) of the 46 Schwannian stroma-poor tumors had high numbers of cancer-associated fibroblasts in the tumor stroma compared to only $2(14 \%)$ of the 14 Schwannian stroma-rich/ dominant tumors $(P<\mathbf{0 . 0 0 1})$. Thirty-three $(\mathbf{8 9} \%)$ of 37 tumors with microvascular proliferation had high numbers of cancer-associated fibroblasts compared to $9(40 \%)$ of 22 tumors without microvascular proliferation $(P<0.001)$. In the xenografts with infiltrating Schwann cells $(n=10)$, the number of cancer-associated fibroblasts per $\mathrm{mm}^{2}$ was approximately sevenfold less than in the control xenografts without stromal Schwann cells $(n=9)$ (mean of $51 \pm 30$ vs $368 \pm 105$, respectively; $P<0.001)$. Thus, cancer-associated fibroblasts were inversely associated with presence of Schwann cells, suggesting that Schwann cells may prevent the activation of fibroblasts. A deeper understanding of the function cancer-associated fibroblasts have in neuroblastoma angiogenesis may guide future development of stroma-directed therapeutic strategies.
\end{abstract}

Modern Pathology (2009) 22, 950-958; doi:10.1038/modpathol.2009.52; published online 1 May 2009

Keywords: cancer-associated fibroblast; neuroblastoma; Schwannian stroma

Cancer-associated fibroblasts are a heterogeneous population of tumor stromal cells that undergo myofibroblastic cytodifferentiation acquiring phenotypic characteristics of activated fibroblasts. ${ }^{1-4}$ In addition to the fibroblast activation protein seprase ${ }^{5}$ and $\alpha$-smooth muscle actin ( $\alpha$-SMA), some cancerassociated fibroblasts express desmin and smooth muscle myosin heavy chain isoforms. ${ }^{1}$ However, they lack expression of high-molecular-weight cal-

Correspondence: Dr SL Cohn, MD, Department of Pediatrics, University of Chicago, 5841 Maryland Avenue, MC 4060, Room N114, Chicago, IL 60637, USA.

E-mail: scohn@peds.bsd.uchicago.edu

Received 8 September 2008; revised 20 February 2009; accepted 23 February 2009; published online 1 May 2009 desmon that distinguishes them from pericytes. ${ }^{6}$ Cancer-associated fibroblast infiltration has been associated with metastasis and poor clinical outcome in colon, ${ }^{7,8}$ breast, $^{9,10}$ prostate ${ }^{11}$ and other adult cancers. ${ }^{12-14}$ The capacity of cancer-associated fibroblasts to promote tumor growth, angiogenesis and invasion is mediated by multiple factors, including hepatocyte growth factor ${ }^{15-17}$ and stromal-cell-derived factor- $1 .^{9}$ However, the function of cancer-associated fibroblasts has not previously been evaluated in neuroblastoma, a pediatric cancer in which clinical behavior is strongly impacted by the cellular composition of tumor stroma.

The current World Health Organization classification of neuroblastoma is based on histological criteria, defined by the International Neuroblastoma 
Pathology Committee (INPC). ${ }^{18-20}$ Four diagnostic categories of Schwannian stroma-rich/dominant tumors include mature and maturing ganglioneuroma, and ganglioneuroblastoma of intermixed and nodular type. Three categories of Schwannian stroma-poor neuroblastoma tumors include differentiated, poorly differentiated and undifferentiated. ${ }^{18,21}$ Tumor aggressiveness is linked to the INPC histological features. ${ }^{18}$ With the exception of nodular ganglioneuroblastoma, all Schwannian stroma-rich neuroblastoma tumors are associated with favorable prognosis. ${ }^{22,23}$ We have previously demonstrated correlation between low vascular density and the absence of microvascular proliferation in Schwannian stroma-rich tumors. ${ }^{24}$ In contrast, high vascular index and abnormal blood vessel structure with microvascular proliferation are associated with clinically aggressive Schwannian stroma-poor neuroblastoma tumors. ${ }^{24,25}$ The mechanisms governing the regulation of angiogenesis and tumor phenotype in neuroblastoma are not fully understood, but we and others have shown that Schwann cells secrete angiogenic inhibitors. ${ }^{26-28}$ In addition, we have demonstrated that angiogenesis is inhibited in a novel neuroblastoma xenograft model in which mouse Schwann cells were induced to infiltrate the tumor by engrafting neuroblastoma cells in the sciatic nerve of nude mice. ${ }^{29}$

To investigate the function cancer-associated fibroblasts have in neuroblastoma angiogenesis and their relationship to stromal Schwann cells, we quantified cancer-associated fibroblasts in 14 Schwannian stroma-rich and 46 Schwannian stroma-poor neuroblastoma tumors. We also quantified the number of cancer-associated fibroblasts in a novel neuroblastoma xenograft model in which murine Schwann cells were induced to infiltrate into the tumor stroma. Similar to previous studies, microvascular proliferation was not detected in Schwannian stroma-rich/dominant neuroblastoma tumors. We also found that high numbers of cancer-associated fibroblasts were inversely associated with the presence of Schwann cells in both the primary human neuroblastoma tumors and in the xenograft model. Our results suggest that cancerassociated fibroblasts have a function in regulating neuroblastoma angiogenesis and that Schwann cells may prevent the accumulation and activation of fibroblasts in neuroblastoma tumors.

\section{Materials and methods}

\section{Patients and Tumor Specimens}

Patients were selected based on the availability of adequate full tissue sections from the primary tumor. Sections from 60 primary neuroblastoma, ganglioneuroblastoma and ganglioneuroma tumors were obtained from Children's Memorial Hospital $(\mathrm{CMH})$ in Chicago at the time of diagnosis, before administration of chemotherapy. Patients were diagnosed between 1986 and 2005. Medical records were reviewed to obtain information regarding patient age, sex, tumor stage, histology, MYCN gene status and outcome. The CMH Institutional Review Board approved this study.

Tumors were grouped into six diagnostic categories recognized by INPC ${ }^{19}$ and staged according to the International Neuroblastoma Staging System. ${ }^{30}$ MYCN gene status was determined in the Children's Oncology Group neuroblastoma Reference Laboratory using FISH. ${ }^{31}$ Tumors were classified as either favorable or unfavorable histology according to the criteria described by INPC. ${ }^{19}$

\section{Cell Culture}

The biological and genetic characteristics of the $M Y C N$-amplified human neuroblastoma cell line, SMS-KCNR, used in this study have been previously described.$^{32}$ Cells were grown at $5 \% \mathrm{CO}_{2}$ in RPMI1640 (Invitrogen, Carlsbad, CA, USA) supplemented with $10 \%$ heat-inactivated fetal bovine serum, Lglutamine and antibiotics.

\section{Sciatic Nerve Engraftment of Neuroblastoma Xenografts}

Sciatic nerve engraftment was carried out as previously described. ${ }^{29}$ Briefly, following anesthesia the left sciatic nerve was surgically exposed and $5 \times 10^{5}$ SMS-KCNR neuroblastoma cells were microinjected either outside or inside the sciatic nerve. Tumors were measured once a week and tumor volume was determined as length $\times$ width $^{2} / 2$. Animals were killed when tumors were $>500 \mathrm{~mm}^{3}$, and the xenografts were harvested and characterized as described by Liu et al. ${ }^{29}$ All animals were treated according to NIH Guidelines for Animal Care and Use, and protocols were approved by the Animal Care and Use Committee at Northwestern University.

\section{Histological Evaluation}

Adjacent $4 \mu \mathrm{m}$ sections from formalin-fixed paraffinembedded tumor tissues were deparaffinized, rehydrated and stained with either hematoxylin and eosin (H\&E) or Masson's Trichrome, ${ }^{33}$ which highlighted variable degrees of fibrocollagenous stroma. The entire area of each section was examined histologically for Schwannian and fibrovascular stroma, vascular morphology and neuroblast differentiation by a pathologist (RZ). A total of 60 human tumors were examined, including 42 neuroblastomas (23 differentiated, 14 poorly differentiated and 5 undifferentiated), 11 ganglioneuroblastomas (7 intermixed and 4 nodular) and 7 ganglioneuromas. Of the neuroblastoma xenografts, 10 were engrafted 
inside the sciatic nerve and 9 were engrafted outside the sciatic nerve and served as controls.

Tumors were classified as having microvascular proliferation when blood vessels were present with thickened walls containing a complete layer of hypertrophic endothelial cells plus additional layers of vascular mural cells as previously described..$^{24}$ The degree of microvascular proliferation varied from slight to florid. ${ }^{24,34}$ Tumors without microvascular proliferation contained only thinwalled vessels with no more than one layer of flat, spindle-shaped endothelial cells. Small vessels forming complex glomeruloid structures were present in tumors with high microvascular proliferation. Special care was taken to avoid confusing tangentially cut vessels. The type of tumor stroma (Schwannian vs fibrovascular) surrounding the vessels was carefully analyzed. Arteries, veins and microvascular proliferation noted within lymph nodes or in intense inflammatory infiltrates were excluded from analysis.

\section{Immunohistochemistry}

Accumulation of cancer-associated fibroblasts in tumors was further evaluated by examining adjacent sections from all human tumors and xenografts stained by immunohistochemistry with $\alpha$-SMA and high-molecular-weight caldesmon. For $\alpha$-SMA staining, $4 \mu \mathrm{m}$ sections were deparaffinized and heatinduced antigen retrieval was carried out in a steamer for $20 \mathrm{~min}$ in Target Retrieval Solution, $\mathrm{pH}$ 9 (DakoCytomation, Carpinteria, CA, USA). For caldesmon staining, deparaffinized sections were treated for $5 \mathrm{~min}$ with Proteinase K (DakoCytomation) at a concentration of $0.04 \mathrm{mg} / \mathrm{ml}$, followed by heat-induced antigen retrieval in a steamer for 20 min in Target Retrieval Solution, pH 6 (DakoCytomation). Slides were immersed in peroxidase block solution (DakoCytomation) and incubated for $1 \mathrm{~h}$ at room temperature with monoclonal mouse antibodies for $\alpha$-SMA (clone 1A4; DakoCytomation) or caldesmon (clone h-CD; DakoCytomation) at 1:50 dilution. Slides were developed with the EnVision + /HRP anti-mouse detection system (DakoCytomation) and counterstained with Gill's hematoxylin.

\section{Quantification of $\alpha$-SMA-Positive Cells in Human Neuroblastoma Tumors}

Tissue sections immunostained for $\alpha$-SMA were scanned at $\times 200$ magnification on the Automated Cellular Imaging System (ACIS II, ChromaVision; Clarient, San Juan Capistrano, CA, USA). To quantify $\alpha$-SMA-positive cells, we defined a protocol for detection by setting color-specific thresholds for both dark and light brown staining using control slides. For each sample, ratios of the areas positively stained for $\alpha$-SMA to the entire area were calculated from the areas provided by ACIS II software and then converted to percentages. The mean percentage of $\alpha$-SMA-positive areas \pm the standard deviation was calculated for histological subsets of neuroblastoma tumors and the Student's $t$-test was used to compare the means. Furthermore, cancer-associated fibroblast number was classified as either low $(\leq 1.0 \%)$ or high $(>1.0)$ based on the percentage of $\alpha$-SMA-positive areas for individual tumors. $\chi^{2}$-Tests were used to compare tumor categories.

\section{Quantification of $\alpha$-SMA-Positive Cells in Neuroblastoma Xenografts}

Cells expressing $\alpha$-SMA were quantified in neuroblastoma xenografts engrafted inside the sciatic nerve and having stromal Schwann cell infiltration and in controls by counting 10 low-power $(\times 200$ magnification) fields. The total number of $\alpha$-SMApositive cells per $\mathrm{mm}^{2}$ was calculated. The mean number of $\alpha$-SMA-positive cells per $\mathrm{mm}^{2}$ in the xenografts with stromal Schwann cell infiltration and controls was compared and statistical significance was analyzed using the Student's $t$-test.

\section{Statistical Analysis}

Associations between cancer-associated fibroblast number and various established clinicopathological prognostic factors of neuroblastoma were analyzed using the $\chi^{2}$-test or Student's $t$-test. Ganglioneuromas were included only in analyses related to stroma histology and microvascular proliferation. Patients were stratified into two risk groups based on stage, age and MYCN status. The non-high-risk

Figure 1 Cancer-associated fibroblasts in Schwannian stroma-poor neuroblastoma tumors. Representative sections of human ganglioneuroma (GNR) (a-d), ganglioneuroblastoma intermixed (GNBI) (e-h), ganglioneuroblastoma nodular (GNBN) (i-l), and neuroblastoma tumors that are differentiated (NBD) ( $\mathbf{m}-\mathbf{p})$, poorly differentiated (NBPD) (q-t) and undifferentiated (NBU) (u-w) tumors stained with H\&E (a, e, i, m, q and $\mathbf{u}$ ), Masson's Trichrome special stain (b, f, j, $\mathbf{n}, \mathbf{r}$ and $\mathbf{v}), \alpha$-SMA that reveals cancer-associated fibroblasts and pericytes (c, $\mathbf{g}, \mathbf{k}, \mathbf{o}, \mathbf{s}$ and $\mathbf{w}$ ) and high-molecular-weight caldesmon, which stains pericytes but not cancer-associated fibroblasts (d, h, l, p and $\mathbf{t}$ ). The mean ( \pm standard deviation) percent of $\alpha$-SMA-positive areas per total tumor area analyzed for each tumor type is shown in the bar graph (x). $\alpha$-SMA-positive, caldesmon-negative cancer-associated fibroblasts are rare in the Schwannian stroma (SS)-dominant ganglioneuroma (c, $\mathbf{d}$ and $\mathbf{x}$ ) and Schwannian stroma-rich ganglioneuroblastoma intermixed (g/h and $\mathbf{x})$ tumors. Significantly more cancer-associated fibroblasts are present in Schwannian stroma-poor tumors (k, l, s, t and w). Bands of fibrocollagenous stroma are thin and delicate in ganglioneuroma and ganglioneuroblastoma intermixed tumors, but are thick and support microvascular proliferation in ganglioneuroblastoma nodular and neuroblastoma tumors (pale blue $\mathbf{b}$ and $\mathbf{f} v s \mathbf{j}$, $\mathbf{n}, \mathbf{r}$ and $\mathbf{v}$ ). Cancer-associated fibroblasts constitute the majority of stromal cells within fibrovascular stroma (o, $\mathbf{s}$ and $\mathbf{w})$. Original magnification $\times 200(\mathbf{a}-\mathbf{l}$ and $\mathbf{t})$ and $\times 400(\mathbf{p})$. 
group included patients with nonamplified $M Y C N$ stage 1, 2 and 3 tumors, and infants with stage 4 and $4 \mathrm{~S}$ neuroblastoma that lacked MYCN amplification.
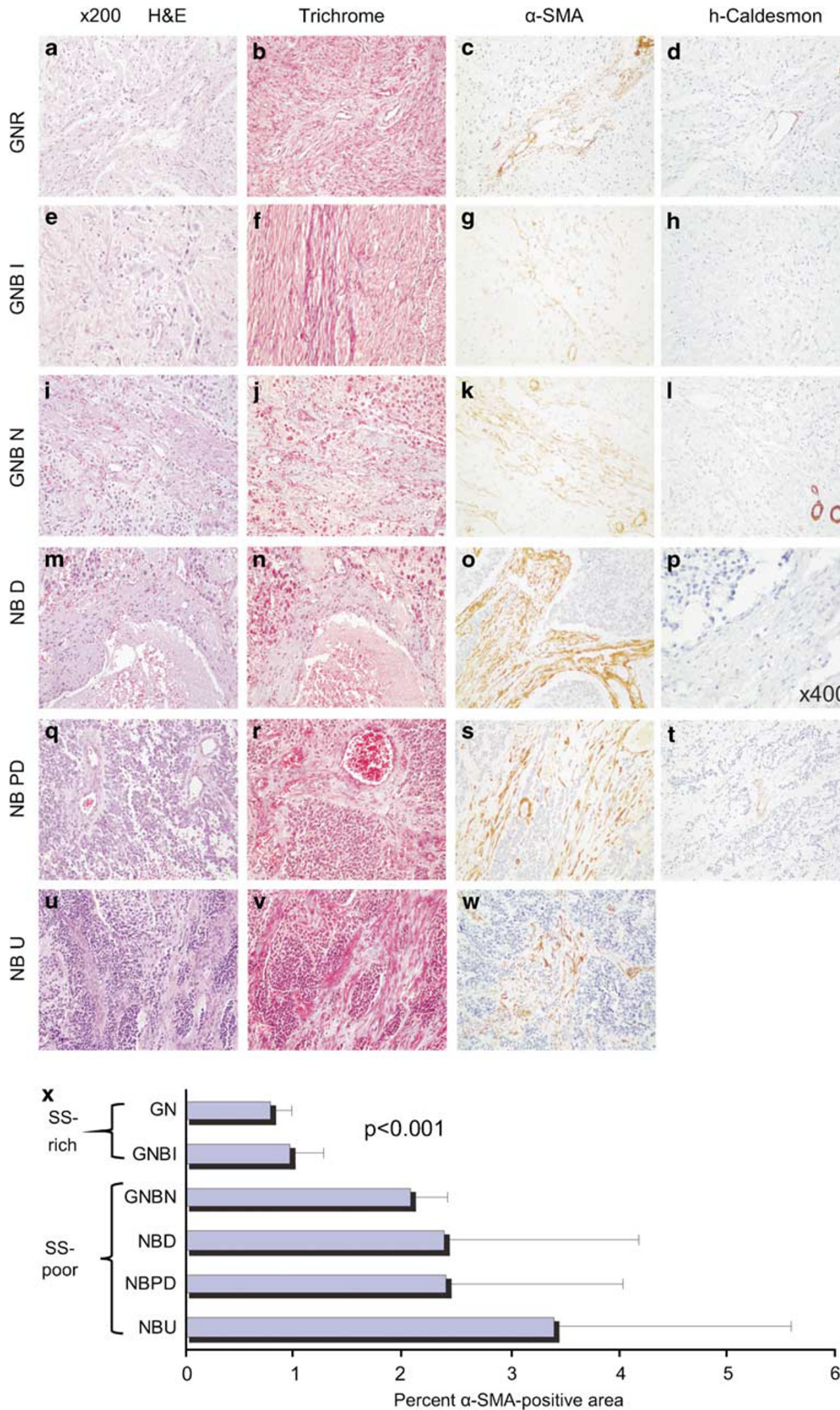

Patients with stage $3 \mathrm{MYCN}$-amplified tumors and children older than 1 year with stage 4 disease were classified as high risk.
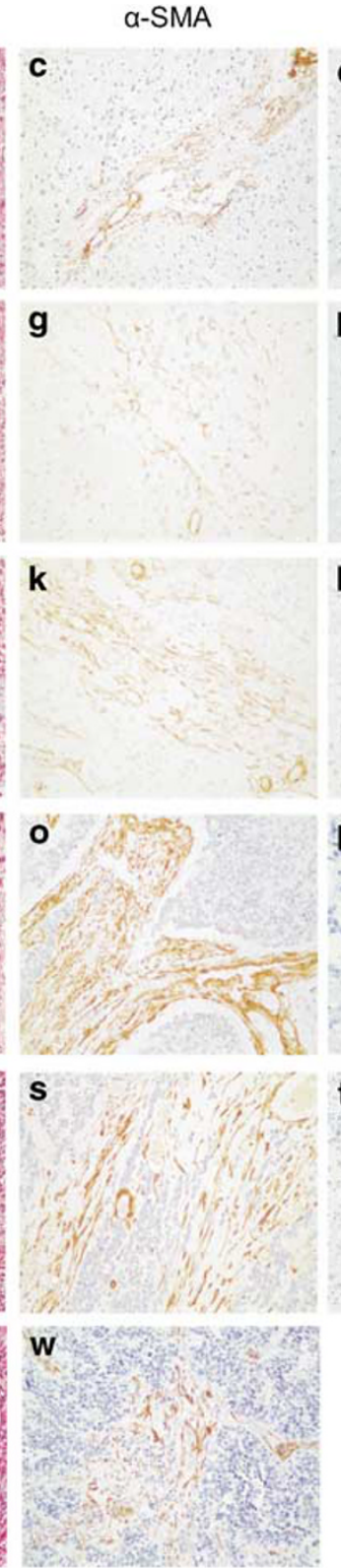

h
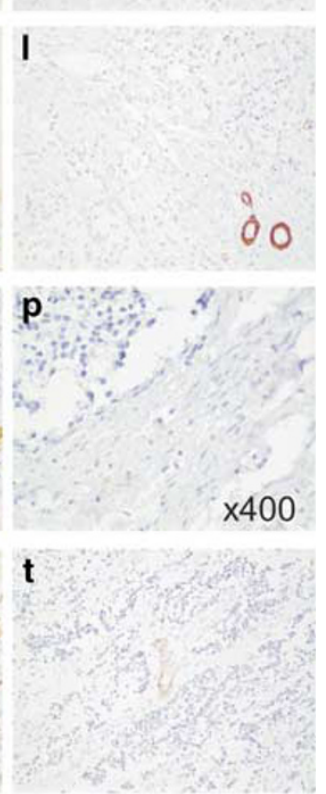

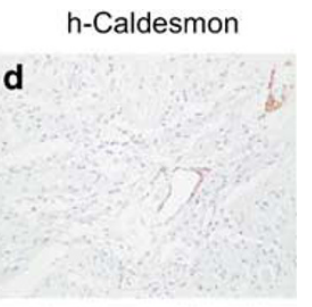




\section{Results}

Cancer-Associated Fibroblast Levels are Associated with Schwannian Stroma-Poor Histology

The vast majority of $\alpha$-SMA-positive cells in the Schwannian stroma-rich/dominant tumors were pericytes as evidenced by their association with endothelial cells and positive h-caldesmon expression. In the ganglioneuroma tumors, scant fibrovascular bands with single layers of pericytes lining thin-walled vessels were seen (Figure 1). Cancerassociated fibroblasts, which express $\alpha$-SMA but not h-caldesmon, were only rarely detected in these tumors. The mean percent $\alpha$-SMA-positive area was low in these Schwannian stroma-dominant tumors, using the defined cutoff value of $\leq 1.0 \%$ $(0.79 \% \pm 0.19$; Figure 1). Similarly, rare scattered cancer-associated fibroblasts were present in the vicinity of maturing neuroblasts and within delicate fibrovascular bands in the ganglioneuroblastoma intermixed tumors. The mean percent $\alpha$-SMA-positive area for the seven ganglioneuroblastoma intermixed tumors was also low $(0.96 \% \pm 0.31)$.

In contrast, staining with $\alpha$-SMA and h-caldesmon demonstrated high numbers of cancer-associated fibroblasts $(\alpha-S M A+/ h C D-)$ in the Schwannian stroma-poor nodules within ganglioneuroblastoma nodular tumors (Table 1). These cells constituted a majority of the stromal cell populations within thick fibrovascular bands and the mean percent $\alpha$-SMA-positive area was high $(2.08 \% \pm 0.34)$. More dramatic increases in cancer-associated fibroblasts were manifested in the Schwannian stroma-poor neuroblastoma tumors and the mean percent $\alpha$ SMA-positive area was high in this subset of tumors $(2.39 \% \pm 1.78$ in differentiated tumors; $2.41 \% \pm 1.64$ in poorly differentiated tumors; $3.40 \% \pm 2.19$ in undifferentiated tumors). Approximately $85-90 \%$ of the stromal cells in the Schwannian stroma-poor

Table 1 Associations between levels of CAFs, Schwannian stroma, MVP and clinical risk factors in the CMH cohort

\begin{tabular}{|c|c|c|c|c|}
\hline Characteristic & CAFs low & CAFs high & Total & $\mathrm{P}$ \\
\hline Diagnosis & & & & $<0.001$ \\
\hline $\mathrm{NB}(\mathrm{A}+\mathrm{B}+\mathrm{C})$ & $9(21 \%)$ & $33(78 \%)$ & 42 & \\
\hline A. Undifferentiated NB & $1(20 \%)$ & $4(80 \%)$ & 5 & \\
\hline B. Poorly differentiated NB & $2(14 \%)$ & $12(86 \%)$ & 14 & \\
\hline C. Differentiated NB & $6(26 \%)$ & $17(74 \%)$ & 23 & \\
\hline Nodular GNB & $0(0 \%)$ & $4(100 \%)$ & 4 & \\
\hline Intermixed GNB & $5(71 \%)$ & $2(29 \%)$ & 7 & \\
\hline GNR & $7(100 \%)$ & $0(0 \%)$ & 7 & \\
\hline \multicolumn{5}{|l|}{ Schwannian stroma } \\
\hline NB+nodular GNB, SS-poor & $9(20 \%)$ & $37(80 \%)$ & 46 & $<0.001$ \\
\hline Intermixed GNB+GNR, SS-rich & $12(86 \%)$ & $2(14 \%)$ & 14 & \\
\hline \multicolumn{5}{|l|}{ Microvascular proliferation } \\
\hline NB+intermixed GNB+GNR, no MVP & $13(60 \%)$ & $9(40 \%)$ & 22 & $<0.001$ \\
\hline NB and nodular GNB with MVP & $5(16 \%)$ & $33(84 \%)$ & 38 & \\
\hline \multicolumn{5}{|l|}{$A g e^{\mathrm{a}}$} \\
\hline$<12$ months & $4(21 \%)$ & $15(79 \%)$ & 19 & 0.51 \\
\hline$\geq 12$ months & $10(29 \%)$ & $24(71 \%)$ & 34 & \\
\hline \multicolumn{5}{|l|}{ Risk group ${ }^{\mathrm{a}}$} \\
\hline High-risk & $3(20 \%)$ & $12(80 \%)$ & 15 & 0.88 \\
\hline Non-high-risk & $7(28 \%)$ & $18(72 \%)$ & 25 & \\
\hline \multicolumn{5}{|l|}{$M Y C N^{\mathrm{a}}$} \\
\hline Amplified & $2(22 \%)$ & $7(78 \%)$ & 9 & 0.75 \\
\hline Nonamplified & $11(28 \%)$ & $29(72 \%)$ & 40 & \\
\hline \multicolumn{5}{|l|}{ Histology ${ }^{\mathrm{a}}$} \\
\hline Unfavorable & $3(13 \%)$ & $20(86 \%)$ & 23 & 0.18 \\
\hline Favorable & $8(29 \%)$ & $20(71 \%)$ & 28 & \\
\hline \multicolumn{5}{|l|}{ Stage $e^{\mathrm{a}}$} \\
\hline $1,2,3,4 \mathrm{~S}$ & $10(29 \%)$ & $24(70 \%)$ & 34 & 0.49 \\
\hline 4 & $3(20 \%)$ & $12(80 \%)$ & 15 & \\
\hline \multicolumn{5}{|l|}{ Survival ${ }^{\mathrm{a}}$} \\
\hline Dead & $2(29 \%)$ & $5(71 \%)$ & 7 & 0.89 \\
\hline Alive & $12(26 \%)$ & $34(74 \%)$ & 46 & \\
\hline
\end{tabular}

GNB: ganglioneuroblastoma; GNR: ganglioneuroma; MVP: microvascular proliferation; NB: neuroblastoma; SS: Schwannian stroma. ${ }^{\mathrm{a}}$ Only NB+GNB. 
neuroblastoma tumors were $\alpha$-SMA positive, either scattered among the neuroblasts or distributed within thick bands of fibrovascular stroma. Masson's Trichrome special stain highlighted fibrocollagenous elements within the fibrovascular stroma of Schwannian stroma-poor neuroblastoma tumors (Figure 1). There is a significant difference of cancer-associated fibroblast levels between Schwannian stroma-rich and Schwannian stromapoor groups $(P<0.001)$, as shown in Figure 1 and Table 1.

\section{Cancer-Associated Fibroblast Levels are Associated with Microvascular Proliferation in Human Neuroblastoma Tumors}

Microvascular proliferation was present in 33 of the 42 Schwannian stroma-poor neuroblastoma tumors and in the stroma-poor nodules of the 4 ganglioneuroblastoma nodular tumors evaluated. In contrast, microvascular proliferation was not detected in the ganglioneuroma and ganglioneuroblastoma intermixed tumors. Of the 22 tumors without microvascular proliferation, $13(68 \%)$ had low cancer-associated fibroblast levels, whereas high numbers of cancer-associated fibroblasts were seen in $33(84 \%)$ of the 38 tumors with microvascular proliferation $(P<0.001)$. Interestingly, cancer-associated fibroblasts were observed in the vicinity of blood vessels with microvascular proliferation (Figure 2).

\section{Heterogeneity of Cancer-Associated Fibroblast Levels in Human Neuroblastoma Tumors}

Table 1 categorizes the extent of cancer-associated fibroblast accumulation according to the available

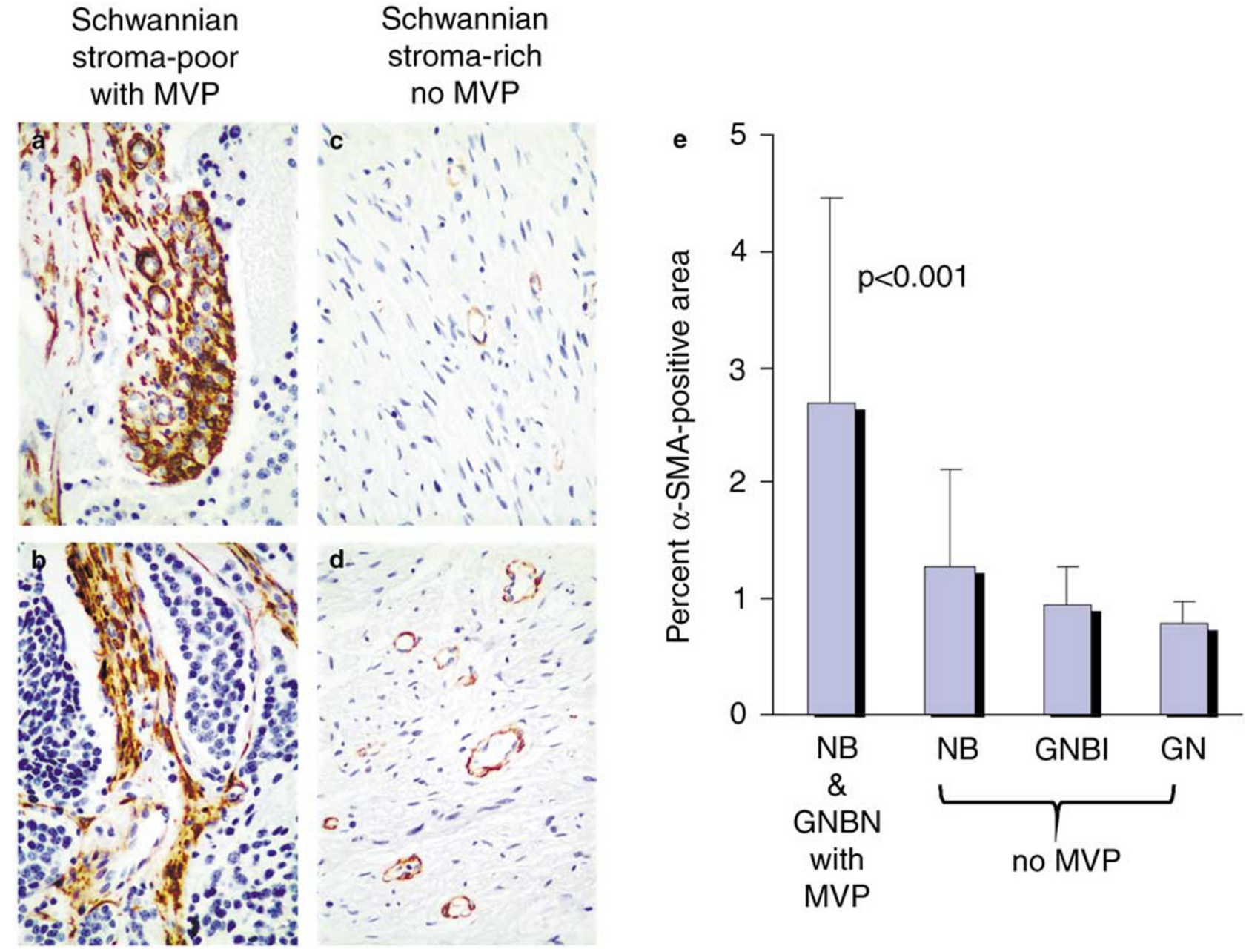

Figure 2 Cancer-associated fibroblasts in human neuroblastomas with microvascular proliferation. Representative sections of differentiated neuroblastoma (NBD) (a), poorly differentiated neuroblastoma (NBPD) (b), ganglioneuroma (GNR) (c) and ganglioneuroblastoma intermixed (GNBI) (d) tumors immunostained for $\alpha$-SMA. The mean ( \pm standard deviation) percent of $\alpha$-SMA-positive areas per total tumor areas analyzed for tumors with microvascular proliferation (MVP) is shown in the bar graph (e). Significantly more $\alpha$-SMA-positive cancer-associated fibroblasts are present in the tumors with microvascular proliferation (a, b and e) as compared to tumors without microvascular proliferation $(\mathbf{c}, \mathbf{d}$ and $\mathbf{e})(P<0.001)$. The cancer-associated fibroblasts are present within bands of fibrovascular stroma supporting microvascular proliferation (a and b). Original magnification $\times 400$. 
clinical and biological characteristics of the human neuroblastoma tumors in the CMH series. Seventeen patients had high-risk disease and 27 patients had non-high-risk disease. The estimated 5-year overall survival for this small cohort of high-risk patients was 61 and $100 \%$ for the subset of non-high-risk children, which is higher than expected. A trend was seen associating presence of cancer-associated fibroblasts with unfavorable histology $(P=0.18)$ but statistical significance was not reached, most likely due to the small number of samples evaluated. In this series of patients, no statistically significant associations were found between high cancer-associated fibroblast levels and $M Y C N$ amplification $(P=0.75)$, age $(P=0.51)$, stage $(P=0.49)$, risk group $(P=0.88)$ or survival $(P=0.89)$.

\section{Cancer-Associated Fibroblast Accumulation is Decreased in Neuroblastoma Xenografts with Schwannian Stroma}

We have previously shown that murine Schwann cells infiltrate the stroma of neuroblastoma xenografts engrafted into the sciatic nerve and that these tumors have inhibited angiogenesis compared to control tumors engrafted outside the sciatic nerve. ${ }^{29}$ Immunohistochemistry with anti- $\alpha$-SMA antibody demonstrated widespread cancer-associated fibroblast infiltration in control neuroblastoma xenografts engrafted outside the sciatic nerve (Figure 3a and b). Interestingly, significantly fewer cancer-associated fibroblasts were detected in the xenografts in which Schwann cells were induced to infiltrate the tumor

\section{Control}
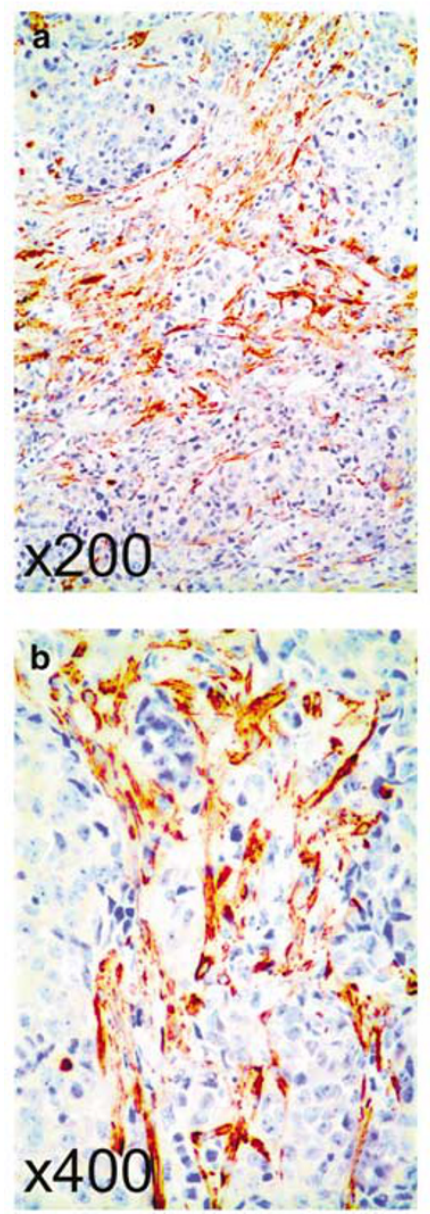

Engrafted inside sciatic nerve
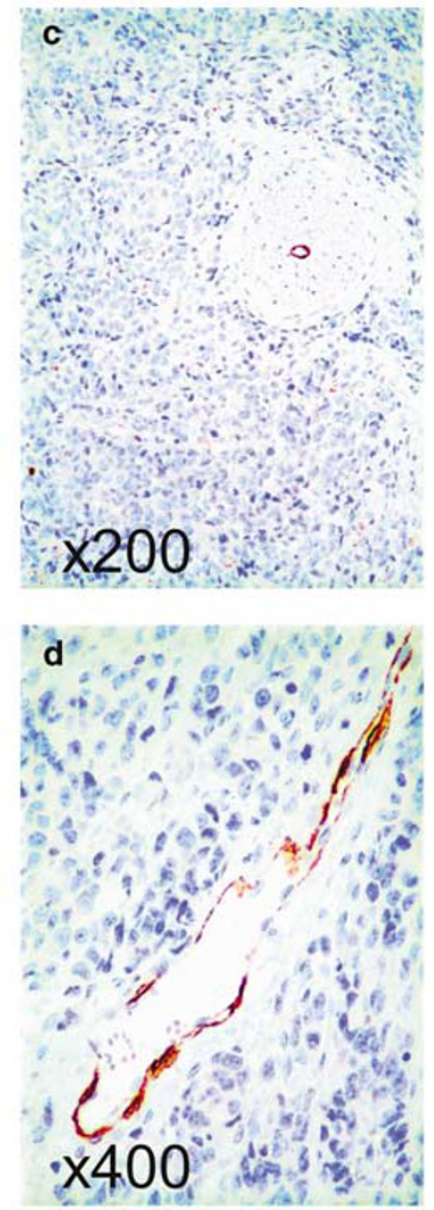

e

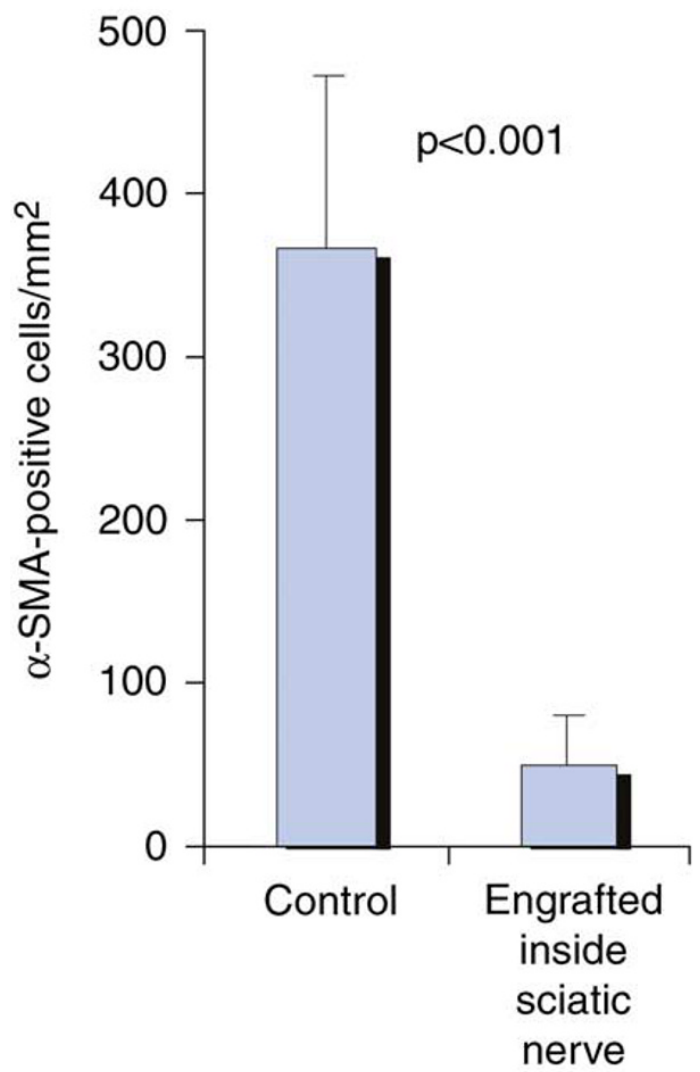

Figure 3 Cancer-associated fibroblasts are decreased in neuroblastoma xenografts with infiltrating murine Schwann cells. Representative sections of SMS-KCNR tumors engrafted outside ( $\mathbf{a}$ and $\mathbf{b}$ ) vs inside (c and $\mathbf{d}$ ) the sciatic nerve immunostained for $\alpha$-SMA. The mean $\left( \pm\right.$ s.d.) number of $\alpha$-SMA-positive cells per $\mathrm{mm}^{2}$ of the tumors engrafted outside $(n=9)$ and inside $(n=10)$ the sciatic nerve is shown in the bar graph (e). Significantly more $\alpha$-SMA-positive cancer-associated fibroblasts are present in tumors engrafted outside (a and b) as compared to inside the sciatic nerve (c and d) $(P<0.001$ for total $\alpha$-SMA-positive cells). Original magnification $\times 200$ (a and $\mathbf{c})$ and $\times 400$ (b and $\mathbf{d})$. 
by engraftment in the sciatic nerve (Figure 3c and d). The number of $\alpha$-SMA-positive cells per $\mathrm{mm}^{2}$ was approximately sevenfold less in xenografts engrafted inside the sciatic nerve $(n=10)$ compared to controls $(51 \pm 30$ vs $368 \pm 105$, respectively; $P<0.001$ ) (Figure 3e).

\section{Discussion}

In neuroblastoma, the amount of Schwannian stroma strongly impacts prognosis, ${ }^{18}$ which has been emphasized in the classification system of Shimada $^{33}$ and the International Neuroblastoma Pathology Classification. ${ }^{19}$ The biological mechanisms that underlie the more benign clinical behavior in tumors with abundant Schwannian stroma are not fully understood. However, we and others have shown that Schwann cells secrete factors that are capable of inducing neuroblastoma differentiation and inhibiting angiogenesis., ${ }^{26,28}$ In contrast to clinically aggressive Schwannian stroma-poor tumors that are highly angiogenic, the vascular index of Schwannian stroma-rich/dominant tumors is low. We have also shown that structurally abnormal blood vessels with microvascular proliferation, which are commonly detected in Schwannian stroma-poor tumors, are rare in Schwannian stroma-rich/dominant neuroblastomas. ${ }^{24}$ We now demonstrate that significantly higher numbers of cancer-associated fibroblasts are present in Schwannian stroma-poor tumors compared to Schwannian stroma-rich/dominant neuroblastomas, consistent with the established pro-angiogenic function of cancer-associated fibroblasts. Although we have previously shown high vascularity to be associated with clinically aggressive disease and poor outcome in neuroblastoma, ${ }^{24,25}$ in the small series of patients analyzed in this study associations between levels of cancer-associated fibroblasts and other established prognostic factors or outcome have not reached statistical significance.

To further explore the seemingly antagonistic relationship between Schwannian stroma and accumulation of cancer-associated fibroblasts, we examined neuroblastoma xenografts in which Schwann cells had been induced to infiltrate into the tumor stroma by surgical engraftment of the neuroblastoma cells within the sciatic nerves of nude mice. ${ }^{29} \mathrm{We}$ had previously documented anti-proliferative and anti-angiogenic effects in these sciatic nerve-engrafted tumors. ${ }^{29} \mathrm{~A}$ dramatic sevenfold decrease in the number of cancer-associated fibroblasts was seen in the neuroblastoma xenografts with stromal Schwann cell infiltration compared to controls. The mechanisms underlying the inverse relationship between high cancer-associated fibroblasts levels and Schwann cells in tumor stroma remain to be elucidated. The altered tumor microenvironment within the sciatic nerve may either have reduced the migration of myofibroblastic precursors into the neuroblastoma xenografts, or inhibited their activation, by one or more Schwann cell-derived factors with tumor suppressive activity. ${ }^{26,28,29}$

Cancer-associated fibroblasts have been identified in breast, colon, lung and prostate cancers, and there is significant evidence demonstrating that these cells actively promote tumor growth and angiogenesis. ${ }^{35,36}$ The origin of tumor myofibroblasts and the underlying mechanisms that lead to their activation are still not well understood. There are reports suggesting that the existing fibroblasts in the tissue stroma can undergo activation. ${ }^{37}$ There is also evidence to suggest that myofibroblasts may originate from the vascular smooth muscles and pericytes. $^{38}$ Studies have shown that transforming growth factor- $\beta$ and platelet-derived growth factor, which are key molecules in the wound healing process, take part in the activation of fibroblasts. ${ }^{13}$ Previous studies from our laboratory have demonstrated that secreted protein acidic and rich in cysteine, an extracellular matrix protein that is secreted by Schwann cells, is capable of preventing fibroblast activation in vitro and in vivo. ${ }^{27}$

Taken together, our results indicate that, similar to other types of carcinoma, cancer-associated fibroblasts promote tumor angiogenesis in neuroblastoma. Furthermore, the paucity of cancer-associated fibroblasts in Schwannian stroma-rich/dominant neuroblastomas that characteristically have low vascularity suggests that Schwann cells may block accumulation and activation of cancer-associated fibroblasts. A deeper understanding of the mechanisms regulating formation of cancer-activated stroma may guide future development of anti-stromal therapeutic strategies in children with aggressive neuroblastoma tumors.

\section{Acknowledgements}

We thank Maria Tretiakova for her assistance with the use of the ACIS II system.

\section{Grant support}

This work was supported by the Neuroblastoma Children's Cancer Society, Friends for Steven Pediatric Cancer Research Fund, the Elise Anderson Neuroblastoma Research Fund, Neuroblastoma Kids and Alex's Lemonade Stand Foundation.

\section{References}

1 Schurch W, Seemayer T, Hinz B, et al. Myofibroblast. In: Mills S (ed). Histology for Pathologists, 3rd edn. Lippincott Williams \& Wilkins: Philadelphia, 2007, pp 123-164.

2 Kalluri R, Zeisberg M. Fibroblasts in cancer. Nat Rev Cancer 2006;6:392-401.

3 Sugimoto H, Mundel TM, Kieran MW, et al. Identification of fibroblast heterogeneity in the tumor microenvironment. Cancer Biol Ther 2006;5:1640-1646. 
4 Desmouliere A, Guyot C, Gabbiani G. The stroma reaction myofibroblast: a key player in the control of tumor cell behavior. Int J Dev Biol 2004;48:509-517.

5 Park JE, Lenter MC, Zimmermann RN, et al. Fibroblast activation protein, a dual specificity serine protease expressed in reactive human tumor stromal fibroblasts. J Biol Chem 1999;274:36505-36512.

6 Nakayama H, Miyazaki E, Enzan H. Differential expression of high molecular weight caldesmon in colorectal pericryptal fibroblasts and tumour stroma. J Clin Pathol 1999;52:785-786.

7 Nakagawa H, Liyanarachchi S, Davuluri RV, et al. Role of cancer-associated stromal fibroblasts in metastatic colon cancer to the liver and their expression profiles. Oncogene 2004;23:7366-7377.

8 Henry LR, Lee HO, Lee JS, et al. Clinical implications of fibroblast activation protein in patients with colon cancer. Clin Cancer Res 2007;13:1736-1741.

9 Orimo A, Gupta PB, Sgroi DC, et al. Stromal fibroblasts present in invasive human breast carcinomas promote tumor growth and angiogenesis through elevated SDF1/CXCL12 secretion. Cell 2005;121:335-348.

10 Yazhou C, Wenlv S, Weidong Z, et al. Clinicopathological significance of stromal myofibroblasts in invasive ductal carcinoma of the breast. Tumour Biol 2004;25:290-295

11 Tuxhorn JA, Ayala GE, Smith MJ, et al. Reactive stroma in human prostate cancer: induction of myofibroblast phenotype and extracellular matrix remodeling. Clin Cancer Res 2002;8:2912-2923.

12 Huber MA, Kraut N, Park JE, et al. Fibroblast activation protein: differential expression and serine protease activity in reactive stromal fibroblasts of melanocytic skin tumors. J Invest Dermatol 2003;120:182-188.

13 Micke P, Ostman A. Tumour-stroma interaction: cancer-associated fibroblasts as novel targets in anti-cancer therapy? Lung Cancer 2004;45(Suppl 2):S163-S175.

14 Chen D, Kennedy A, Wang JY, et al. Activation of EDTA-resistant gelatinases in malignant human tumors. Cancer Res 2006;66:9977-9985.

15 Nakamura T, Matsumoto K, Kiritoshi A, et al. Induction of hepatocyte growth factor in fibroblasts by tumor-derived factors affects invasive growth of tumor cells: in vitro analysis of tumor-stromal interactions. Cancer Res 1997;57:3305-3313.

16 Martin TA, Parr C, Davies G, et al. Growth and angiogenesis of human breast cancer in a nude mouse tumour model is reduced by NK4, a HGF/SF antagonist. Carcinogenesis 2003;24:1317-1323.

17 Davies G, Mason MD, Martin TA, et al. The HGF/SF antagonist NK4 reverses fibroblast- and HGF-induced prostate tumor growth and angiogenesis in vivo. Int J Cancer 2003;106:348-354.

18 Schwab M, Shimada H, Joshi V, et al. Neuroblastic tumours of adrenal gland and sympathetic nervous system. In: Kleihues P, Cavanee WK (eds). Pathology and Genetics, Tumours of the Nervous System. IARC Press: Lyon, 2000, pp 153-161.

19 Shimada H, Ambros IM, Dehner LP, et al. The International Neuroblastoma Pathology Classification (the Shimada system). Cancer 1999;86:364-372.

20 Shimada H, Ambros IM, Dehner LP, et al. Terminology and morphologic criteria of neuroblastic tumors: recommendations by the International Neuroblastoma Pathology Committee. Cancer 1999;86:349-363.
21 Qualman SJ, Bowen J, Fitzgibbons PL, et al. Protocol for the examination of specimens from patients with neuroblastoma and related neuroblastic tumors. Arch Pathol Lab Med 2005;129:874-883.

22 Nagoshi M, Tsuneyoshi M, Enjoji M. S-100 positive undifferentiated neuroblastomas with a special reference to the tumor stroma related to favorable prognosis. Pathol Res Pract 1992;188:273-283.

23 Hachitanda Y, Nakagawara A, Nagoshi M, et al. Prognostic value of N-myc oncogene amplification and S-100 protein positivity in children with neuroblastic tumors. Acta Pathol Jpn 1992;42:639-644.

24 Peddinti R, Zeine R, Luca $\mathrm{D}$, et al. Prominent microvascular proliferation in clinically aggressive neuroblastoma. Clin Cancer Res 2007;13:3499-3506.

25 Meitar D, Crawford SE, Rademaker AW, et al. Tumor angiogenesis correlates with metastatic disease, N-myc amplification, and poor outcome in human neuroblastoma. J Clin Oncol 1996;14:405-414.

26 Chlenski A, Liu S, Crawford SE, et al. SPARC is a key Schwannian-derived inhibitor controlling neuroblastoma tumor angiogenesis. Cancer Res 2002;62:7357-7363.

27 Chlenski A, Guerrero LJ, Yang Q, et al. SPARC enhances tumor stroma formation and prevents fibroblast activation. Oncogene 2007;26:4513-4522.

28 Crawford SE, Stellmach V, Ranalli M, et al. Pigment epithelium-derived factor (PEDF) in neuroblastoma: a multifunctional mediator of Schwann cell antitumor activity. J Cell Sci 2001;114:4421-4428.

29 Liu S, Tian Y, Chlenski A, et al. Cross-talk between Schwann cells and neuroblasts influences the biology of neuroblastoma xenografts. Am J Pathol 2005;166: 891-900.

30 Brodeur GM, Seeger RC, Barrett A, et al. International criteria for diagnosis, staging, and response to treatment in patients with neuroblastoma. J Clin Oncol 1988;6:1874-1881.

31 Mathew P, Valentine MB, Bowman LC, et al. Detection of MYCN gene amplification in neuroblastoma by fluorescence in situ hybridization: a pediatric oncology group study. Neoplasia 2001;3:105-109.

32 Yang QW, Liu S, Tian Y, et al. Methylation-associated silencing of the thrombospondin-1 gene in human neuroblastoma. Cancer Res 2003;63:6299-6310.

33 Shimada H, Chatten J, Newton Jr WA, et al. Histopathologic prognostic factors in neuroblastic tumors: definition of subtypes of ganglioneuroblastoma and an age-linked classification of neuroblastomas. J Natl Cancer Inst 1984;73:405-416.

34 Rojiani AM, Dorovini-Zis K. Glomeruloid vascular structures in glioblastoma multiforme: an immunohistochemical and ultrastructural study. J Neurosurg 1996;85:1078-1084.

35 Tuxhorn JA, McAlhany SJ, Dang TD, et al. Stromal cells promote angiogenesis and growth of human prostate tumors in a differential reactive stroma (DRS) xenograft model. Cancer Res 2002;62:3298-3307.

36 De WO, Mareel M. Role of tissue stroma in cancer cell invasion. J Pathol 2003;200:429-447.

37 Ronnov-Jessen L, Van DB, Celis JE, et al. Smooth muscle differentiation in cultured human breast gland stromal cells. Lab Invest 1990;63:532-543.

38 Skalli O, Pelte MF, Peclet MC, et al. Alpha-smooth muscle actin, a differentiation marker of smooth muscle cells, is present in microfilamentous bundles of pericytes. J Histochem Cytochem 1989;37:315-321. 